\title{
LEY APLICABLE AL RÉGIMEN ECONÓMICO MATRIMONIAL, A PROPÓSITO DEL COMENTARIO DE LA SENTENCIA DE LAAUDIENCIA PROVINCIAL DE MADRID, DE 30 SEPTIEMBRE 2019*
}

\author{
APPLICABLE LAW TO MATRIMONIAL PROPERTY REGIME, \\ WITH OCCASION OF COMMENTARY OF THE JUDGMENT \\ OF THE APPEAL COURT OF MADRID, 30TH SEPTEMBER 2019*
}

\author{
JULIANA RODRÍGUEZ RODRIGO \\ Profesora Titular de Derecho Internacional Privado \\ Universidad Carlos III de Madrid \\ ORCID ID: 0000-0002-5896-983X
}

Recibido: 03.03.2020 / Aceptado: 27.03.2020

DOI: https://doi.org/10.20318/cdt.2020.5662

\begin{abstract}
Resumen: La Audiencia Provincial de Madrid ha resuelto, con esta sentencia, el recurso de apelación planteado ante ella, por la inclusión de determinados bienes en el inventario realizado con ocasión de la liquidación del régimen económico matrimonial. La pareja litigante posee nacionalidad neerlandesa y, en primera instancia, la esposa probó el Derecho de los Países Bajos, sin oposición del marido, como ordenamiento aplicable a la cuestión. El esposo recurre alegando la aplicación del Derecho español para excluir determinados bienes del reparto. El órgano judicial español, en el marco del artículo 9.2 $\mathrm{CC}$, concluye que la legislación aplicable es la de los Países Bajos, por la concurrencia del primer punto de conexión del precepto, por la nacionalidad común de los cónyuges en el momento de la celebración del matrimonio.
\end{abstract}

Palabras clave: régimen económico matrimonial, Reglamento 2016/1103, artículo 9.2 CC, prueba del Derecho extranjero, reenvío.

Abstract: The Appeal Court of Madrid has resolved the brought sue due the inclusion of specific assets in the inventory. The couple has Dutch nationality and, in the first instance, the wife proved Netherlands law, without opposition from her husband. The husband appeals alleging the application of Spanish law in order to exclude certain assets from the inventory. The Spanish judge, within the framework of article $9.2 \mathrm{CC}$, considers the Netherland law is the applicable legislation.

Keywords: matrimonial property regime, Regulation 2016/1103, article 9.2 CC, foreign law proof, renvoi.

Sumario: I. Hechos del caso; II. Reglamento 2016/1103; III. Normas de producción interna españolas; 1. Artículos 9.2 y 9.3 CC; 2. Artículo 9.2 CC; 3. Prueba del Derecho extranjero; IV. Reenvío; 1 . Definición y clases; 2 . Artículo 12.2 CC; 3 . Convenio de La Haya sobre ley aplicable a los regímenes matrimoniales, de 14 de marzo de 1978; 4. Admisión del reenvío; V. Conclusiones.

"Este trabajo ha sido realizado en el marco del Proyecto de investigación "Los principios del Derecho Romano en el Derecho europeo del siglo XXI - Programa Logos Fundación BBVA de Ayudas a la Investigación en el Área de Estudios Clásicos" 2020-2022". 


\section{Hechos del caso}

1. El asunto, del que trae causa la resolución de la Audiencia Provincial de Madrid objeto de comentario, es un litigio por la formación del inventario que se ha de repartir tras el proceso de divorcio de las partes. El recurrente -el marido- reclama que tres de los bienes que ha considerado como comunes el Juzgado de Primera Instancia de Torrejón de Ardoz, no lo son y, por lo tanto, pide que se excluyan del inventario. Para ello, el apelante alega el Derecho español, como ley aplicable al régimen económico matrimonial. La esposa, por su parte, indica que el Derecho neerlandés, aplicado en primera instancia, no fue objeto de impugnación por parte del ahora actor y que, por ello, este debe ser el ordenamiento regulador de la cuestión.

2. La Audiencia Provincial de Madrid, en lo que a este trabajo interesa, se cuestiona si el Derecho español es el aplicable, tal como alega el recurrente.

\section{Reglamento 2016/1103}

3. El órgano judicial no se plantea en ningún momento aplicar el Reglamento 2016/1103, por el que se establece una cooperación reforzada en el ámbito de la competencia, la ley aplicable, el reconocimiento y la ejecución de resoluciones en materia de regímenes económicos matrimoniales ${ }^{1}$. Esta norma europea ha empezado a aplicarse a partir del 29 de enero de 2019, si bien, cuando se trata de determinar el Derecho rector de la materia en cuestión, sólo se aplica si el matrimonio se ha celebrado después de esa fecha señalada -o cuando los cónyuges han realizado una elección de ley a partir de ese momento- (art. 69.3) ${ }^{2}$.

En nuestro caso, no se cuestiona la competencia judicial internacional. Es la ley aplicable la que es objeto de controversia, por esta razón, el Reglamento no puede tenerse en cuenta, ya que, las partes en el litigio contrajeron matrimonio en el año 1995, fecha anterior al 29 de enero de 2019, y, según se desprende de la sentencia comentada, no ha habido capitulaciones matrimoniales. Por todo ello, si bien la respuesta de la Audiencia Provincial de Madrid es correcta en este punto, no habría estado de más que el órgano judicial hubiera mencionado la existencia de esta norma europea para, a continuación, indicar su no aplicación en el caso ${ }^{3}$.

\footnotetext{
${ }^{1}$ DOUE L183, de 8 julio 2016

${ }^{2}$ H. PÉroz/E. Fongaro, Droit international privé patrimonial de la famille, $2^{\mathrm{a}}$ ed., Lexis Nexis, Paris, 2017, pp. 125-126; I. BARRIÈRE BrousSE, "Le patrimoine des couples internationaux dans l'espace judiciaire européen. Les règlements européens du 24 juin 2016 relatifs aux régimes matrimoniaux et aux effets patrimoniaux des partenariats enregistrés", JDI Clunet, $\mathrm{n}^{\circ} 2$, 2017, p. 493; L. Usunier, "Libre, mobile, divers: le couple au miroir du droit international privé de l'Union européenne", Revue Trimestrielle de droit civil, $\mathrm{n}^{\circ}$ 4, 2016, pp. 808-809; C. Nourissat/M. ReviLlard, "Règlements européens du 24 juin 2016 sur les régimes matrimoniaux et les effets patrimoniaux des partenariats enregistrés", Defrénois, no 17, 15 septembre 2016, p. 883; N. JOUBERT, "La dernière pierre (provisoire?) à l'édifice du droit international privé europeén en matière familiale. Les règlements du 24 juin 2016 sur les régimes matrimoniaux et les effets patrimoniaux des partenariats enregistrés”, RCDIP, n 1, 2017, p. 5; B. AÑoveros terradAs, "El régimen conflictual de las capitulaciones en los nuevos Reglamentos de la Unión Europea en materia de regímenes económicos matrimoniales y efectos patrimoniales de las uniones registradas", Anuario Español de Derecho Internacional Privado, t. XVII, 2017, p. 832; P. WAUTELET, "What's Wrong with Article 22? The Unsolved Mysteries of Choice of Law for Matrimonial Property”, Yearbook of private international law, vol. XIX, 2017-2018, p. 223.

No se aplicará, por tanto, a elecciones de ley realizadas antes del 29 de enero de 2019, aún cuando las partes hayan acordado que tengan efectos después de esa fecha (D. Coester-Waltjen, "Connecting Factors to Determine the Law Applicable to Matrimonial Property Regimes", Yearbook of private international law, vol. XIX, 2017-2018, p. 196).

${ }^{3}$ En estos supuestos, el órgano judicial competente deberá aplicar las normas de conflicto existentes en su ordenamiento -los artículos 9.2 y $9.3 \mathrm{CC}$, en el caso español- (L. UsuNIER, "Libre, mobile, divers: le couple au miroir du droit international privé de l'Union européenne", Revue Trimestrielle de droit civil, $\mathrm{n}^{\circ}$ 4, 2016, p. 809; E. Gallant, "Le nouveau droit international privé européen des régimes patrimoniaux de couples", Europe, mars 2017, p. 7). Por ello, serán muchos los casos en los que, a partir del 29 de enero de 2019, los jueces españoles sigan aplicando estos preceptos 9.2 y 9.3 CC para determinar la ley reguladora del régimen patrimonial de la pareja. Y, éste, objeto de la sentencia de la Audiencia Provincial de Madrid, es uno de ellos.
} 


\section{Normas de producción interna españolas}

\section{Artículos 9.2 y $9.3 \mathrm{CC}$}

4. Una vez que se ha descartado la aplicación del Reglamento 2016/1103 en este caso, el órgano judicial competente debe acudir a sus normas de producción interna para determinar el Derecho aplicable al régimen económico matrimonial de la pareja. Estas normas de conflicto españolas son los artículos 9.2 y 9.3 del Código Civil.

5. El artículo $9.2 \mathrm{CC}$ dispone que "los efectos del matrimonio se regirán por la ley personal común de los cónyuges al tiempo de contraerlo; en defecto de esta ley, por la ley personal o de la residencia habitual de cualquiera de ellos, elegida por ambos en documento auténtico otorgado antes de la celebración del matrimonio; a falta de esta elección, por la ley de la residencia habitual común inmediatamente posterior a la celebración, y, a falta de dicha residencia, por la ley del lugar de celebración del matrimonio".

Por su parte, el artículo 9.3 CC indica que "los pactos o capitulaciones por los que se estipule, modifique o sustituya el régimen económico del matrimonio serán válidos cuando sean conformes bien a la ley que rija los efectos del matrimonio, bien a la ley de la nacionalidad o de la residencia habitual de cualquiera de las partes al tiempo del otorgamiento".

6. La relación entre ambos preceptos consiste en que el artículo 9.2 CC se va a aplicar a los efectos económicos del matrimonio -además de a los efectos personales- y el artículo $9.3 \mathrm{CC}$ puede ser utilizado en relación con estos cuando las partes otorguen capitulaciones matrimoniales ${ }^{4}$. Por lo tanto, el artículo 9.2 CC proporciona un único ordenamiento para todos los efectos del matrimonio. Así es, en esta materia existe el principio de unidad ley, según el cual, todos los efectos van a quedar regulados por la misma normativa, la determinada por el artículo $9.2 \mathrm{CC}$; salvo que las partes decidan que su régimen económico matrimonial quede regulado por otra ley en virtud del artículo $9.3 \mathrm{CC}$.

7. Debemos distinguir, por tanto, dos situaciones. Por un lado, cuando las partes no pactan nada en relación con los efectos patrimoniales, el artículo 9.2 CC se va a ocupar de determinar el régimen económico matrimonial legal. Por otro lado, si existen capitulaciones matrimoniales, el artículo $9.2 \mathrm{CC}$ sería el aplicable y, si la ley a la que lleva este precepto no da validez a los pactos económicos hechos por las partes, se debería acudir al resto de conexiones que ofrece el artículo $9.3 \mathrm{CC}^{5}$. Dicho con otras palabras, ambos preceptos confluyen cuando se trata de determinar la ley aplicable al régimen económico matrimonial pactado. En este caso, la relación entre ellos es de jerarquía, primero se aplicará el artículo 9.2 y, después, si según este artículo las capitulaciones matrimoniales no son válidas, se podrá acudir al precepto $9.3^{6}$.

\footnotetext{
${ }^{4}$ Con la entrada en vigor del Reglamento 650/2012, la ley sucesoria se aplica, también, a los derechos sucesorios del cónyuge viudo y, por tanto, la remisión que realiza el artículo $9.8 \mathrm{CC}$ a la aplicación del Derecho rector del régimen económico matrimonial para los derechos sucesorios del contrayente supérstite, ya no puede tenerse en cuenta.

Reglamento (UE) $n^{\circ}$ 650/2012, del Parlamento Europeo y del Consejo, de 4 de julio de 2012, relativo a la competencia, la ley aplicable, el reconocimiento y la ejecución de las resoluciones, a la aceptación y la ejecución de los documentos públicos en materia de sucesiones mortis causa y a la creación de un certificado sucesorio europeo, DO 201, de 27 julio 2012.

${ }^{5}$ En contra, P. QuinzÁ Redondo, "Inscripción en el Registro de la Propiedad de una escritura de compraventa bajo régimen económico-matrimonial extranjero: La DGRN de 10 de mayo de 2017”, Bitácora Millennium DIPr., núm. 6, 2017, p. 5. Para este autor, el artículo $9.3 \mathrm{CC}$ se aplica cuando existe régimen económico matrimonial pactado y el 9.2 cuando no lo hay.

${ }^{6}$ A.L. Calvo Caravaca/J. Carrascosa González, "Efectos del matrimonio", en A.L. Calvo Caravaca/J. Carrascosa GonZÁLEZ (dirs.), Derecho Internacional Privado, vol. II, $17^{\mathrm{a}}$ ed., Comares, Granada, 2017, pp. 236-239; RDGRN de 13 noviembre 2015, FD III y IV (JUR 2016\115973).

En contra, M. AMORES CONRADI, para quien "actualmente la diferencia de contenido entre los $n^{\circ} 2$ y 3 del art. 9 no se encuentra en que se refieran a distintos conceptos, pues el supuesto del $\mathrm{n}^{\circ} 3$ no es sino una fracción del de su precedente, sino en que se refieren a hipótesis distintas: que haya o no pacto específico de los cónyuges sobre su régimen económico matrimonial. Con esta ordenación, y aunque el orden seguido por el legislador sea al opuesto, lo primero que debe examinarse es si existe o no pacto entre los cónyuges, si tiene validez y con qué límites, su régimen jurídico y sólo después procederá examinar la norma más general, que viene a cumplir una función subsidiaria" (M. AMORES CONRADI, "La nueva ordenación de la ley aplicable a los efectos del matrimo-
} 


\section{Artículo 9.2 CC}

8. En el caso que nos ocupa, la Audiencia Provincial de Madrid directamente acude al artículo 9.2 CC, sin hacer ninguna referencia al hecho de si la pareja había acordado o no la ley aplicable a su régimen económico matrimonial. Debemos entender, por tanto, que no existe ese pacto de elección de ley y que, por ello, se trata de determinar la regulación del régimen económico matrimonial legal en este supuesto.

Bien, presuponiendo que no existen capitulaciones matrimoniales, el órgano judicial español competente no debe plantearse qué precepto del artículo 9 CC debe aplicar en este caso, si el 9.2 o el 9.3, está claro que es el primero el único posible a tener en cuenta.

9. Según este artículo, la primera conexión aplicable para determinar el Derecho rector del régimen económico matrimonial es la nacionalidad común de los cónyuges en el momento de la celebración de la unión conyugal. En realidad, el artículo $9.2 \mathrm{CC}$ alude a la ley personal común, sin embargo, esta ley se corresponde con la de la nacionalidad común, según indica el precepto $9.1 \mathrm{CC}^{7}$. Esta ley nacional común es la que tengan en el momento de la celebración del matrimonio y no la que pudieran obtener con motivo del mismo ${ }^{8}$.

10. La conexión nacionalidad presenta ventajas e inconvenientes ${ }^{9}$. En relación con las primeras, la nacionalidad permite que la ley aplicable no cambie con facilidad y que, por tanto, la ley reguladora del régimen económico matrimonial a través de esta conexión sea estable en el tiempo. Efectivamente, el domicilio o la residencia habitual cambian con mayor frecuencia que la nacionalidad, por ello, como conexión estable, la nacionalidad es la más adecuada. Además de lo anterior, la ley nacional refleja la vinculación que siempre el legislador español ha realizado entre el régimen económico matrimonial y el estatuto personal ${ }^{10}$. Por último, dentro de las ventajas, se considera que la conexión nacionalidad favorece a los países de emigración, ya que, la ley nacional se seguirá aplicando más allá de las fronteras nacionales a los ciudadanos que han emigrado. La conexión residencia habitual se presenta más adecuada que la nacionalidad para los países receptores de inmigrantes. De esta forma, con la ley de la residencia habitual se aplicará la ley del foro a los extranjeros que residan en el país y, también, a los nacionales que se encuentran establecidos en el territorio.

Por el lado de los inconvenientes, el más importante es el que acontece cuando la conexión lleva a un país totalmente desvinculado de la relación jurídica litigiosa ${ }^{11}$. Esto es, la nacionalidad puede atri-

nio", Revista Jurídica de Castilla La Mancha, enero-agosto 1991, n 11-12, p. 43). Para este autor la explicación de que el artículo 9.3 CC remita a la ley de los efectos se encuentra en que, a través del primero, los cónyuges pueden hacer uso de la autonomía de la voluntad material y elaborar pactos que serán válidos si la ley de los efectos les da validez (vid., en este sentido, P. DiAGo DiAGO, Pactos o capitulaciones matrimoniales en Derecho internacional privado, El Justicia de Aragón, Zaragoza, 1999, pp. 228-229).

7 SAP de Barcelona, de 22 marzo 2010, ECLI:ES:APB:2010:2765; SAP de Huesca, de 14 diciembre 2005 (AC 2005\2377).

8 A.L. Calvo Caravaca/J. Carrascosa González, "Efectos del matrimonio", en A.L. Calvo Caravaca/J. Carrascosa GonZÁLEZ (dirs.), Derecho Internacional Privado, vol. II, ed. 17ª, Comares, Granada, 2017, p. 227.

9 A favor de la conexión nacionalidad común, vid., M. Agullar Benítez De Lugo, "Los efectos del matrimonio", en M. Aguilar Benitez de Lugo y otros, Lecciones de Derecho civil internacional, Tecnos, Madrid, 1996, p. 153. En contra de esta conexión para los efectos de matrimonio, vid., A.L. Calvo Caravaca/J. Carrascosa González, "Efectos del matrimonio", en A.L. Calvo Caravaca/J. Carrascosa GonzÁlez (dirs.), Derecho Internacional Privado, vol. II, ed. 17", Comares, Granada, 2017, p. 227; J. Pirrung, "La réforme du droit international privé en République fédérale d'Allemagne spécialment dans le domaine des relations entre époux", Travaux du Comité français de droit international privé, 1986/1987, pp. 201-222; A. BoRRÁs RodríguEz, "Non discrimination à raison du sexe et modification du droit international privé espagnol", Revue Critique de Droit International Privé, 1991, vol. LXXX, pp. 626-634; ID., "No discriminación por razón de sexo: Derecho internacional privado español", Anuario de Derecho Civil, enero-marzo 1991, tomo XLIV 44, pp. 233-249; M. Amores ConRADI, “Arts. 9.2 y 9.3 Cc", R. Bercovitz Rodríguez Cano, (coord.), Comentarios a las reformas del Código civil (Desde la ley 21/1987 de 11 de noviembre a la ley 30/1991, de 20 de diciembre), Tecnos, Madrid, 1993, pp. 4474-456.

${ }^{10}$ E. Rodríguez Pineau, Régimen económico matrimonial: aspectos internacionales, Comares, Granada, 2002, p. 30.

11 A.L. Calvo Caravaca/J. Carrascosa González, "Efectos del matrimonio", en A.L. Calvo Caravaca/J. Carrascosa GonzÁLEz (dirs.), Derecho Internacional Privado, vol. II, ed. 17a, Comares, Granada, 2017, p. 227. 
buirse por un hecho anecdótico y realmente no representar ninguna conexión de los sujetos con ese país cuya nacionalidad tienen. En esos casos, en supuestos de cónyuges que poseen la nacionalidad de un país con el que no tienen ninguna relación, esta conexión no cumpliría con el principio de previsibilidad en la ley aplicable que inspira las normas de conflicto ${ }^{12}$.

11. Además de lo anterior, otro inconveniente es la dificultad de determinar la nacionalidad en determinadas situaciones. Así, ad ex., en los casos de múltiple nacionalidad, de apatridia, de nacionalidad indeterminada o en los supuestos en los que algún cónyuge -o los dos- son refugiados. En estos casos, el órgano judicial tendrá que establecer previamente qué ley debe tener en cuenta para saber si concurre el punto de conexión de la nacionalidad común.

En nuestro caso no hay ninguna duda al respecto, nuestro supuesto no es ninguno en los que se podrían encontrar problemas para determinar la nacionalidad de los cónyuges. Tal como recoge la Audiencia Provincial de Madrid, las dos partes en el litigio tienen nacionalidad neerlandesa. Esta nacionalidad es la que tenían en el momento de contraer el matrimonio y es la que siguen conservando en la actualidad. Todo ello, pese a haber residido 10 años en España -en el momento del recurso, ambos han vuelto a residir en Países Bajos-.

12. Como conclusión a todo lo anterior, siguiendo lo que indica el artículo $9.2 \mathrm{CC}$, la ley aplicable al régimen económico matrimonial de la pareja es el Derecho de los Países Bajos.

\section{Prueba del Derecho extranjero}

13. Una vez que se determina que el Derecho aplicable al recurso planteado es el neerlandés, corresponde ahora estudiar la prueba del Derecho extranjero.

14. La Audiencia Provincial de Madrid no cuestiona la prueba que realiza la parte actora cuando presenta la demanda ante el Juzgado de Primera Instancia. El órgano judicial de apelación simplemente transcribe casi todas las normas aplicables en este punto y concluye que la esposa prueba el Derecho neerlandés, sin oposición del marido. Por lo tanto, es el ordenamiento de los Países Bajos el aplicable en el caso.

15. Decimos que el órgano judicial competente transcribe casi todas las normas reguladoras de la prueba del Derecho extranjero porque falta el tratamiento de la carga de la prueba del Derecho extranjero. En este sentido, el artículo 282 LEC se ocupa de establecer la regla general de que las partes son las que tienen que probar el Derecho extranjero. Y, en nuestro caso, como son ellas las que lo prueban, no hay ningún problema relacionado con este aspecto y, por ello, el tribunal acepta la prueba del Derecho neerlandés que aporta la esposa y que no cuestiona el marido.

\section{Reenvío}

16. La Audiencia Provincial de Madrid, sin embargo, no se plantea la posibilidad de aceptar el reenvío que pudiera realizar el Derecho neerlandés al ordenamiento español.

\section{Definición y clases}

17. El reenvío supone aplicar la norma de conflicto del ordenamiento extranjero al que conduce la norma de conflicto del foro. Esto se debe al principio de aplicación integral del Derecho extranjero,

\footnotetext{
${ }^{12}$ Así se puso de manifiesto en la SJPI núm 1 de Badalona, de 15 abril 1988 y en la SAP Barcelona de 6 abril 1989 (vid., N. Bouza VidAL, "Nota a la SJPI núm 1 de Badalona de 15 abril 1988 y SAP Barcelona (Sección 13) de 6 abril 1989”, Revista Española de Derecho Internacional, 1990, vol. XLII, pp. 264-271).
} 
según el cual, la norma de conflicto del foro remite al entero ordenamiento jurídico extranjero, no sólo a las normas materiales, también a las conflictuales, y, por ello, como el supuesto de hecho es internacional, la norma aplicable en ese Derecho extranjero debe ser otra norma de conflicto ${ }^{13}$.

18. Además de lo anterior, la razón de ser del reenvío se encuentra en el clásico principio de Derecho Internacional Privado de la armonía internacional de soluciones ${ }^{14}$. Esto es, con esta figura se pretende, y se consigue, que el Derecho material aplicable al caso sea el mismo independientemente del juez que conozca del mismo, sea el del foro o sea el del Estado extranjero cuya norma de conflicto materializa el reenvío.

19. Existen dos clases de reenvío. Podemos hablar del reenvío de primer grado y del reenvío de segundo grado.

- Reenvío de primer grado o de retorno. Este tipo de reenvío se produce cuando la norma de conflicto extranjera remite la regulación de la situación jurídica al Derecho del foro. De esta forma, el juez competente aplicará finalmente, si se acepta el reenvío, el Derecho propio.

- Reenvío de segundo grado o reenvío ulterior. En este caso, la norma de conflicto extranjera tiene un punto de conexión que indica que el Derecho aplicable a la cuestión es el de un tercer Estado. Cuando el reenvío no es de retorno se debe poner límite a las sucesivas remisiones que pueden realizar las normas de conflicto, ya que, de lo contrario, podría no concretarse el Derecho material aplicable por los múltiples reenvíos entre normas de conflicto que pueden encadenarse -reenvío de tercer grado, de cuarto, etc.--

\section{Artículo 12.2 CC}

20. Puesto que nos encontramos en el marco de las normas de producción interna españolas, la regulación del reenvío a tener en cuenta es la recogida en el artículo 12.2 CC. Según este precepto, " $L a$ remisión al derecho extranjero se entenderá hecha a su ley material, sin tener en cuenta el reenvio que sus normas de conflicto puedan hacer a otra ley que no sea la española". Esto es, el reenvío admitido en España es únicamente el de retorno. El artículo 12.2 CC, con redacción confusa eso sí, ha sido interpretado en este sentido de permitir que en España se acepte únicamente el reenvío que la norma de conflicto extranjera realice al ordenamiento español.

No obstante lo anterior, es cierto que en nuestro país convivimos con la Ley cambiaria y del cheque, que constituye una excepción al reenvío de retorno puesto que permite el reenvío de segundo grado $^{15}$. Esta contradicción con el artículo 12.2 CC se debe a que esta Ley fue una copia ciega del llamado Derecho cambiario ginebrino ${ }^{16}$.

\footnotetext{
13 A.L. Calvo Caravaca/J. Carrascosa GonzÁlez, Derecho Internacional Privado, vol. I, $18^{a}$ ed., Comares Granada, 2018 , p. 308

${ }^{14}$ P. DE CESARI, Autonomia della volontà e legge regolatrice delle successioni, Padova, Cedam, 2001, p. 140; D. DAMASCELLI, "I criteri di collegamnto impiegati dal regolamnto n.650/2012 per la designazione della legge regolatrice della successione a causa di morte", en P. FRANZINA/A. LEANDRO (a curda di), Il diritto internazionale privato europeo delle successioni mortis causa, Consiglio nazionale del notariado Collana di Studi, 2013, Giuffrè, Milano, p. 97; J. Carrascosa GonzÁlez, El Reglamento Sucesorio Europeo 650/2012 de 4 de julio 2012. Análisis crítico, Comares, Granada, 2014, pp. 279-280.

Esta es la ventaja que menciona A. BonOMI acerca del reenvío. En cuanto a los inconvenientes, afirma que la complejidad en su aplicación es la principal razón negativa que podría aducirse al reenvío (A. Bonomi, "Il regolamento europeo sulle successioni”, RDIPP, 2013, p. 307).

En relación con este clásico principio de Derecho Internacional Privado, vid., M.-P. WeLLER, “Anknüpfungsprinzipien im Europäischen Kollissionsrecht: Abschid von der “Klassischen”IPR-Dogmatik?”, IPRax, 5, 2011, pp. 429-437.

${ }^{15}$ Ley 19/1985, de 16 de julio, cambiaria y del cheque, BOE núm. 172, de 19 julio 1985.

16 A.L. Calvo Caravaca/J. Carrascosa González, Derecho Internacional Privado, vol. I, $18^{\mathrm{a}}$ ed., Comares, Granada, 2018, p. 246.
} 
21. En virtud de este precepto $12.2 \mathrm{CC}$, el órgano judicial español podría plantearse la aplicación del Derecho nacional si la norma de conflicto neerlandesa remitiese al ordenamiento español.

\section{Convenio de La Haya sobre ley aplicable a los regímenes matrimoniales, de 14 de marzo de 1978}

22. La norma de conflicto de los Países Bajos en esta materia de regímenes matrimoniales es el Convenio de La Haya sobre ley aplicable a los regímenes matrimoniales, de 14 de marzo de $1978^{17}$. Esta norma convencional es aplicable a los matrimonios celebrados a partir del 1 de septiembre de 1992 y hasta el 28 de enero de $2019^{18}$. La unión conyugal objeto de nuestro litigio se encuentra cubierta por la norma, ya que, como hemos indicado antes, se celebró en 1995.

23. Según este texto convencional, en defecto de elección de ley, el ordenamiento aplicable a los regímenes económico matrimoniales es el del Estado de la residencia habitual común después del enlace (art. 4). No obstante, en determinadas circunstancias previstas en el artículo 5, la ley aplicable será la de la nacionalidad común de los cónyuges (art. 4). Y, en defecto de residencia habitual común tras el matrimonio y de nacionalidad común, el ordenamiento del Estado que presente los vínculos más estrechos con la relación controvertida (art. 4).

24. Países Bajos ha realizado la declaración que contempla el artículo 5 , segundo párrafo, 1) del Convenio de La Haya y, por ello, concurre una de las circunstancias previstas en el precepto mencionado para que la ley aplicable sea la de la nacionalidad común, neerlandesa, de los cónyuges. Por ello, en el caso en cuestión, el órgano judicial español no podría plantearse la admisión del reenvío, ya que, la norma de conflicto extranjera no indica que debe aplicarse el Derecho español en el caso.

\section{Admisión del reenvío}

25. No obstante lo anterior, parece conveniente señalar que, si este fuera el supuesto, esto es, si el Convenio de La Haya remitiera a la legislación española, el órgano judicial nacional podría admitir el reenvío en determinadas situaciones ${ }^{19}$.

26. Para empezar, el artículo $12.2 \mathrm{CC}$ no es obligatorio, es facultativo. Tal como indica el TS, " $\mathrm{La}$ frase «tener en cuenta» no equivale a que deban aplicarse nuestras normas sucesorias de modo inexorable, puesto que en español (Diccionario de la Real Academia) «tener en cuenta» quiere decir "tener presente, considerar», y ello es lo que debe hacer esta Sala: considerar si el reenvío (que ya ha sido alguna vez admitido por nuestros Tribunales de instancia) ha de aceptarse en el presente caso, [...]"20.

27. En segundo lugar, el reenvío no puede suponer una vulneración de los principios fundamentales que inspiran la norma de conflicto en cuestión. Así es, "El artículo 12.2 contiene una norma de carácter general, de las denominadas por la doctrina «norma de aplicación o funcionamiento» que no puede interpretarse aisladamente, sino en relación con la norma especifica y concreta que regula la materia $[\ldots]^{21}$. En este sentido, uno de los principios que inspira la regulación conflictual del régimen económico matrimonial en España es la unidad de ley. Tal como hemos explicado anteriormente, el artí-

\footnotetext{
${ }^{17}$ https://assets.hcch.net/docs/3fccda38-481c-4bf1-b41b-b07fc5346654.pdf

${ }^{18}$ La fecha de 28 de enero de 2019 se debe a que Países Bajos es parte del Reglamento 2016/1103.

${ }^{19}$ STS de 15 noviembre 1996, RJ 1996\8212; STS 21 mayo 1999, RJ 1999\4580; STS 23 septiembre 2002, RJ 2002\8029;STS 12 enero 2015, ECLI:ES:TS:2015:422.

${ }^{20}$ STS de 15 noviembre 1996, RJ 199618212.

${ }^{21}$ STS de 15 noviembre 1996, RJ 199618212.
} 
culo 9.2 CC indica el ordenamiento aplicable a todos los efectos del matrimonio, tanto a los económicos como a los personales, imponiendo, así, la misma y única normativa a todos ellos ${ }^{22}$.

En nuestro caso, de admitirse el reenvío, no se produciría una vulneración del principio de unidad de ley, puesto que, si fuera el supuesto, el juez español aplicaría el ordenamiento nacional a todos los bienes que forman parte del inventario de la pareja. El Convenio de La Haya no regula diferentes leyes para distintas cuestiones relativas al régimen económico matrimonial; salvo que las partes elijan ordenamiento aplicable, en este caso, sí pueden escoger la ley del país de situación de los bienes, para los inmuebles (art. 3). En nuestro supuesto, como partimos del hecho de que los cónyuges no han realizado capitulaciones matrimoniales, la ley aplicable a todos los bienes de la pareja es la misma, la ley de la residencia habitual común después del matrimonio, la ley de la nacionalidad común o la ley del país que presenta los vínculos más estrechos con el caso, dependiendo de las circunstancias.

28. En tercer lugar, además de lo anterior, el reenvío debe conducir a resultados justos para el ordenamiento español. En palabras del TS, "Con relación al contexto argumentativo de la parte recurrente debe señalarse, en primer término, que no puede desconocerse que la reciente doctrina jurisprudencial de esta Sala, si por tal entendemos la desarrollada a partir de a Sentencia de 15 de noviembre de 1996, ha flexibilizado la aplicación meramente literal del reenvio, tal y como se contempla en el artículo 12.2 (donde el reenvío de retorno solo se acepta en favor del ordenamiento jurídico español), afirmando la necesidad ab initio (desde el inicio) de ponderar su efectiva aplicación a la concurrencia de otros criterios. Entre estos, se ha señalado la conveniencia de que el reenví lleve a un resultado acorde con los principios generales del Derecho español de forma que, en su caso, entrañe una mayor justicia en relación a los intereses en juego (STS de 21 de mayo de 1999). Pero sobre todo, caso que nos ocupa, se ha destacado el valor referencial que desarrolla la figura del reenvío como instrumento al servicio de la armonización de sistemas jurídicos de los Estados (la ya citada STS de 15 de noviembre de 1996) ${ }^{23}$.

Por lo tanto, el reenvío debe ser coherente con la armonía internacional de soluciones. Este concepto, como ya se ha mencionado, significa que, independientemente del tribunal que conozca del caso, la ley material aplicable debe ser la misma. Sin embargo, el Tribunal Supremo español lo interpreta de forma diferente $\mathrm{y}$, en este sentido, considera que hay armonía internacional de soluciones cuando los principios en los que se fundamenta la legislación del Estado extranjero en la materia son similares a los del Estado del foro. En el caso de sucesiones, por ejemplo, el TS entiende que no existe armonía internacional de soluciones cuando en el Derecho extranjero existe la libertad de testar mientras que en España existen las legítimas y, por lo tanto, no concurre esa libertad absoluta de disposición para el causante ${ }^{24}$.

Desde el momento en que España y los Países Bajos forman parte del mismo Reglamento en materia de régimen económico matrimonial, la armonía internacional de soluciones se respetaría, ya que, ambos Estados deben compartir los mismos principios en esta materia, principios recogidos en la norma europea de la que ambos son parte.

29. Por todo lo dicho, la Audiencia Provincial de Madrid podría haber admitido el reenvío -si lo hubiera habido- y haber aplicado el Derecho español en el caso.

\footnotetext{
22 En relación con las sucesiones: “La negación del reenvío puede apoyarse en que la colisión entre el estatuto sucesorio El derecho español entiende preponderante la Ley Nacional del «de cuius»; al propio tiempo, el sistema hereditario español es de carácter universalista, esto es, sostiene el criterio de unidad del régimen sucesorio” (STS de 15 noviembre. RJ 1996 18212 ). 23 STS 12 enero 2015, ECLI:ES:TS:2015:422

${ }^{24}$ El reenvio, por último, debe entenderse como un instrumento de armonización de sistemas juridicos de los Estados, como un instrumento respetuoso con los principios que los inspiran y si el derecho americano se apoya en una gran libertad de testar, y no reconoce las legítimas de los hijos, en nada armonizaría la coexistencia de los derechos respectivos, la aplicación por esta Sala del derecho español a la sucesión del causante de este litigio, que no ha conservado con España ni la residencia ni el domicilio" (STS de 15 noviembre 1996, RJ 1996\8212).
} 


\section{Conclusiones}

30. La sentencia objeto de comentario no reviste ninguna complicación. Se trata de un supuesto de hecho fácil de resolver, sin aristas que haya que limar.

31. En el caso en cuestión, un matrimonio de neerlandeses se divorcia y litigan por los bienes que han de formar parte del inventario resultante de la liquidación del régimen económico de la pareja. El matrimonio se celebró en el año 1995 y, por ello, el órgano judicial español no puede aplicar el Reglamento 2016/1103 para determinar la ley aplicable. En el marco del artículo $9.2 \mathrm{CC}$, por lo tanto, y, en defecto de capitulaciones matrimoniales, el ordenamiento rector es el de la nacionalidad común de los cónyuges en el momento de la celebración del matrimonio; la ley de los Países Bajos. Este Derecho extranjero es probado por la esposa, sin oposición del marido. Por todo ello, el juez español aplica dicho ordenamiento y resuelve la demanda.

32. Si bien no plantea ningún problema, la resolución de apelación ha permitido poder analizar la aplicación del Reglamento europeo sobre régimen económico matrimonial, la regulación española de la prueba del Derecho extranjero y la posibilidad de admisión del reenvío en el caso, cuestiones, todas ellas, interesantes y que podrían tener que resolverse en otros supuestos de régimen económico matrimonial que pudieran plantearse ante autoridades españolas. 